\title{
Pragmatic Trade-offs in Utterance-Based Systems: Uncovering Technological Implications
}

\author{
Kathleen F. McCoy \\ Department of Computer and Information Sciences \\ University of Delaware \\ mccoy@cis.udel.edu \\ Jan L. Bedrosian \\ Department of Speech Pathology and Audiology \\ Western Michigan University \\ jan.bedrosian@wmich.edu \\ Linda A. Hoag \\ Program in Communication Sciences and Disorders \\ Kansas State University \\ hoag@humec.ksu.edu
}

\section{Introduction}

Advances in technology have begun to make an impact on Augmentative and Alternative Communication (AAC) system technology in particular. For example, utterance-based systems that provide reasonably easy access to prestored messages for conversation are becoming a reality. In using these systems, the user must anticipate text needs and store appropriate messages in his/her device to be called up when needed during a conversation. However, because it is impossible to anticipate the exact conversational context in which a prestored message might be needed, it will be the case that some prestored messages will not be appropriate for the discourse context in which they are needed. The user must then make a decision concerning whether it is better to quickly deliver a message that is inappropriate with respect to the conversational context, or to take the time, possibly several minutes, to edit the prestored message to make it contextually appropriate. The user must choose, then, between the contextual appropriateness of the message and the speed at which that message is delivered. In either case, the choice is not perfect.

We contend that the answer to this dilemma is not straightforward. The conventional wisdom among AAC researchers (Creech, 1996a, 1996b; Grant 1995; Higginbotham \& Wilkins, 1999; Todman \& Alm, 1997) seems to be "longer is better" - that is, that AAC users are judged to be more communicatively competent when they use longer messages. However, these findings pertain to longer messages that are completely appropriate for the conversational context in which they are generated. In our work we are concerned with the relationship between the message and the conversational context. This has not previously been addressed. Thus, the question remains as to what happens when prestored text is used that involves messages that are not entirely appropriate.

As technology developers of the next generation of AAC systems (which are likely to contain some sort of utterance-based technology), we must be informed by user and partner preferences and theories that explain the outcomes of these preferences. Unfortunately, AAC is not a theorydriven field of study (Grove, Clibbens, Barnett, \& Loncke, 1998). While there has been some 
research that identifies variables relevant to successful interaction between AAC system users and their typically speaking partners (e.g., Bedrosian, Hoag, Calculator, \& Molineux, 1992; Hoag and Bedrosian, 1992; Light, Beer, Buchert, Casey, DiMarco, \& Dolan, 1995), we have only a fragmented and incomplete picture. This is especially the case where the use of utterance-based technology is involved.

At the same time, technology is expensive to develop and the price of making inappropriate design decisions is great (e.g., a costly device that does not have the features desirable to the user and is therefore left on the shelf to collect dust). Thus, in addressing technological needs in future systems, technology designers must be guided by theory and research. Our research team is conducting a series of experiments to help understand conversational tradeoffs, between the contextual appropriateness of messages and their speed of message delivery, encountered by users of future utterance-based systems. Once a theory has been built, we propose that it be used to guide developers of AAC technology in providing options that best support prestored text usage consistent with that theory.

\section{Rise in Research on Utterance-Based Text Systems}

Advances in utterance-based technology, such as Talk Boards (Mayer-Johnson, Co.), are beginning to open up new possibilities for AAC systems (Alm, Morrison, \& Arnott, 1995; Todman \& Alm, 1997; Vanderheyden, 1995a, 1995b). The need for these systems is clear: using prestored text enables significantly faster communication.

Major issues to date in utterance-based systems have centered around enabling users to quickly and easily (i.e., with minimal cognitive effort) retrieve the prestored text message that they feel is appropriate for the given discourse situation. This is no doubt a crucial research issue. Utterancebased systems could not work if appropriate access to the prestored messages cannot be achieved. This is not, however, the end of the story. People developing technology in this area have (understandably) focused on retrieving topically appropriate messages - with little to no regard to how these messages might fit into the current discourse context. A theory of conversational tradeoffs in AAC device use might help guide the technology to appropriately scaffold user needs.

\section{What Situations should we Focus on?}

There is no perfect AAC system. Users have different requirements in different situations. So, what is the setting in which one should develop a theory pertaining to conversations using utterance-based systems?

In our research, we have chosen to investigate a situation involving an AAC user in a public setting transacting normal business (e.g., going to a bookstore). There are two aspects of this setting that make it appealing. First, this setting is a public one where the people interacting with the AAC user are unfamiliar with the devices and the individuals who use them. Often people involved in AAC technology get information from parents, teachers, and others who have a personal investment in communicating with particular AAC users. These people have a vested interest in solving everyday problems involving the AAC user. However, consider that communicating with such familiar individuals is very different from communicating in a more public setting. Familiar people bring a wealth of knowledge that makes up much of the context for communication with the AAC user. The AAC user and these familiar partners may construct personal and idiosyncratic means of communication, but these routines cannot be expected to work 
with others. Consider the importance of communication with unfamiliar partners in a public setting for AAC users. A key to independence is the ability to independently communicate with people that the AAC user does not know and who cannot be expected to be familiar with AAC devices and strategies. Thus, what we are interested in are the reactions of these unfamiliar communication partners so we can determine what strategies work with them.

A second aspect of the bookstore setting is that it appears to be a setting well suited for using utterance-based technology because:

- It may be reasonable to anticipate the many text needs in this setting, but difficult to anticipate the exact context in which they might be needed. A mismatch could lead to misinterpretation by the sales clerk making it difficult to complete the task at hand.

- Speed is important. In a public situation such as purchasing items in a bookstore, speed pressure exists in that other customers are likely waiting, and the sales clerk may be in a hurry to move these people through the line.

- Grammatical appropriateness is important. When communicating with friends in private, it is often quite reasonable to use less-than-perfect grammatical structures. Among friends it is the message that is important and not necessarily the medium (i.e., syntactic structure) through which it is delivered. Friends are familiar with the AAC device and can work to figure out meanings. In a public setting, however, when dealing with strangers, it is important that messages be grammatically clear.

- Impressions are important. If the sales clerk has a negative impression (e.g., he/she is scared, anxious) then that sales clerk may not provide the service to the customer that is needed. For example, the AAC customer may be asked to step aside, or the sales clerk may just give the user any item to get him/her out of the store.

In our series of experiments we will be examining the effects of tradeoffs between the contextual appropriateness of messages and the speed of delivery of these messages on sales clerks' attitudes toward AAC users and their communication. The ultimate goal is to determine which are the best conversational choices so that technology to support those choices can be developed.

\section{How can prestored messages be contextually ill-formed?}

Our aim is to develop a theory of conversational tradeoffs for the AAC users who employ utterance-based systems. In order to do this, we chose to first consider an existing theory governing typical conversations. We turned to Grice's (1975) theoretical framework in order to identify ways in which a prestored message might not fit the particular conversational context.

Grice claims that speakers in a conversation follow the following Cooperative Principle:

Make your conversational contribution such as is required, at the stage at which it occurs, by the accepted purpose or direction of the talk exchange in which you are engaged. (Grice, 1975, p. 45).

He goes on to further specify a set of conversational maxims or rules falling into 4 categories that explain what it means to be cooperative.

Categories of Maxims: 
- Quantity - contributions to a conversation should contain no more and no less information than is required for the purposes of the exchange

- Quality - contributions to a conversation should be true, and based on adequate evidence

- Relation - contributions to a conversation should be relevant

- Manner - contributions to a conversation should avoid obscurity of expression and ambiguity, and should be brief and orderly

Grice claims that speakers and listeners share an implicit knowledge of these maxims, and that speakers draw upon this shared knowledge to convey meaning. For instance, in a situation where one person has asked where a gallon of milk can be purchased, a response of "There is a convenience store around the corner" can be interpreted as more than a simple statement of a fact about the location of a convenience store. The response also implies (1) the store carries milk, and (2) the store is open. In conversations, the speaker may flaunt one or more of the maxims by generating an utterance that does not follow a maxim. When the listener recognizes such flaunting, he/she automatically looks for a non-conventional interpretation.

Grice's theory offers an explanation of how utterance meaning can be derived from interpreting the utterance in a particular conversational context. At the same time, it can be used to characterize ways in which an existing prestored message may not exactly match up with the conversational context in which an AAC user may find him/herself at the time such a message is delivered. Such a message may be flawed in that it may violate one or more of the maxims. It is not at all clear how a listener would interpret such a message, nor what effect it would have on the conversation. Note that Grice did not propose his cooperative principle with AAC system users in mind. We propose that the framework provided by Grice's theory could be modified to examine outcomes, as measured by public attitudes, when the communicator cannot comply with the rules and must choose which ones to violate.

We used three of the categories of maxims to characterize ways in which a prestored message may be contextually inappropriate: (1) Quantity - an existing prestored message may contain excessive information (i.e., more information than is necessary for the purpose of the exchange) or inadequate information (i.e., less information than is appropriate for the purpose of the exchange), (2) Relation - a prestored message may contain some information that is not relevant to the current situation, and (3) Manner - a message may contain some redundant information. The alternative to delivering a prestored message that is ill-formed in one of the above ways is to edit the message to fix it. This editing would take extra time, which might also violate the maxim of manner. According to Grice, messages should be delivered with reasonable dispatch.

\section{Prestored Text Situations with Maxim Violations Involving Tradeoffs between Message Appropriateness and Speed}

In our experiments, we are studying violations of these maxims in videotaped scripted conditions taking place at the checkout counter of a bookstore. Our subjects, who are currently employed as sales clerks, are asked to project themselves into the role of the clerk in the store as they are viewing the conditions. In a situation such as a bookstore, it is quite reasonable that the user may not able to exactly anticipate the text needs. Consider how this could lead to prestored messages that violate Grice's maxims. 


\section{Quantity}

Recall that the maxim of quantity states that the message should contain no more or no less information than is required. Suppose our AAC user has been perusing Amazon.com and has run across and downloaded the description of a book s/he desires from the web page. This description not only contains the author and title of the book, but also contains additional information such as the price, number of pages, ISBN number, and copyright date. Delivering such a message would violate the maxim of quantity as it contains more information than is necessary for the purpose of the exchange (the title and author alone are sufficient to identify the book).

The question is: is it better for the user to quickly deliver this pragmatically odd message quickly, or to take the time to edit the message for contextual appropriateness (where that extra time is likely a violation of the maxim of manner)? What are the attitudes of the naive subjects in these different situations?

Consider that a message may also contain too little information. For example, suppose an AAC user hears that a new Stephen King book is out, but does not know the book's title. In this situation s/he might prestore a generic message such as "I'd like the Stephen King book please." Once s/he arrives at the bookstore (and sees the sales posters around) s/he COULD edit the information to add in the book's title - but the user is in a hurry and thus doesn't want to take the time to edit it before reaching the front of the line. Should s/he use the stored message or take the time to construct the more complete message?

\section{Relevance}

The user may have a message that contains some information that is irrelevant to the current situation. Consider the following possibility. Suppose the user downloads headlines from a newspaper to use in a current events discussion group. Further suppose that one of the downloaded headlines is about Stephen King (one of the user's favorite authors). It might read, "Stephen King was seriously injured in a ht and run accident." Now suppose that one day, while at the shopping mall, the user decides on the spur of the moment that s/he would really like to buy the new Stephen King book that had just come out. The problem is that s/he had not previously anticipated stopping at the bookstore, and so s/he does not have a prestored message that is completely appropriate for this situation. Rather, the only message stored on his/her device that could quickly be accessed is the line: Stephen King was seriously injured in a hit-and-run accident. Should s/he use this message (since it does contain the author's name) in the hope that the clerk will be able to figure out what the intended meaning is? The other choice would be to edit the message, but that would take additional time causing the message to not be delivered with reasonable dispatch (required by the maxim of manner). What will the clerk think with respect to either of these messages?

\section{Manner}

A third area concerns the maxim of manner. In our experiments, a prestored message may violate the maxim of manner if it is no brief and orderly because it contains repetition. Note that the maxim of manner may also be violated because the user may choose to either construct a new message from scratch or to edit an existing message to make it fit the current discourse context. The additional time taken for constructing/editing will violate this maxim as well.

Let's consider how a prestored message may be redundant or repetitious. Consider a situation where a user wants to purchase several separate books and has stored a request for each of the 
books as a separate message just in case s/he needs to go to more than one bookstore to find all of the books. For example, for each of the books s/he might store "I'd like you to help me find <title and author > in paperback please." However, when the user gets up to the counter, s/he realizes that s/he should ask the sales clerk for all of the books at the same time - yet each of the stored utterances would repeat all of the words except for the title and author. What will the sales clerk think about this rather repetitious request? How will it affect the attitudes of the sales clerk?

\section{Current Status: Experiments}

Our preliminary research has validated a set of scripts containing violations of Grice's maxims as discussed. Subjects recognize the particular violations we wish to investigate in the scripts we have developed. Experiments are currently being conducted to determine the effects of tradeoffs between message appropriateness and speed of delivery on attitudes toward the AAC user. From the findings, we hope to develop appropriate technologies to support the user's effective communication in an expeditious fashion.

Potential technological developments that might be required include:

- technology to make editing of prestored messages very fast

- technology that allows a message to be quickly pieced together from a set of partial phrases

- $\quad$ suggestions for storing and then piecing prestored message segments together to form contextually appropriate pieces. The pieces of the messages may be related to the previously specified maxims.

- If violations of the maxims do not appear to affect attitudes, future technology might focus on retrieving topically appropriate messages (and provide little support for editing or piecing together at the time of message delivery)

\section{Acknowledgments}

This work has been supported by Department of Health and Human Services, National Institutes Of Health, National Institute on Deafness and Other Communication Disorders, Grant Number: 1 R01 DC03670-02.

\section{References}

Alm, N., Morrison, A., \& Arnott. L. (1995). A communication system based on scripts, plans, and goals for enabling nonspeaking people to conduct telephone conversations. Proceedings of IEEE Systems, Man and Cybernetics, 2408-2412.

Bedrosian, J. L., Hoag, L., Calculator, S. N., \& Molineux, B. (1992). Variables influencing perceptions of the communicative competence of an adult augmentative and alternative communication system user. Journal of Speech-Language and Hearing Research, 35, 1105-1113.

Creech, R. (1996a) Extemporaneous speaking: Pragmatic principles. Paper Presented at the 4th Annual Pittsburgh Employment Conference, Pittsburgh, PA.

Creech, R. (1996b) Speaker's bureau: System operators. Paper Presented at the Minspeak Conference, Wooster, $\mathrm{OH}$. 
Grant, S. (1995) Using TALK.Communication Matters, 9, 22-25.

Grice, H. P. (1975). Logic and conversation. In P. Cole \& J. L. Morgan (Eds.), Syntax and semantics, Volume 3; speech acts, (pp. 41-58), New York: Academic Press.

Grove, N., Clibbens, J., Barnett, S., \& Loncke, F. (1998). Constructing theoretical models of augmentative and alternative communication. In E. Bjorck-Akesson \& P. Lindsay (Eds.), Communication naturally: Theoretical and methodological issues in augmentative and alternative communication, (48-64). Proceedings of the Fourth ISAAC Research Symposium.

Hoag, L. \& Bedrosian, J. L. (1992). Effects of speech output type, message length, and reauditorization on perceptions of the communicative competence of adult augmentative and alternative communication system user. Journal of Speech and Hearing Research, 35, 1363-1366.

Higginbotham, J. D. \& Wilkins, D. P.(1999). Slipping through the timestream: Social issues of time and timing in augmented interactions. In D. Kovarsky, J. Duchan, \& M. Maxwell, (Eds.), Constructing (in)competence: Disabling evaluations in clinical and social interaction, Mahwah, New Jersey: Lawrence Erlbaum Associates.

Light, J., Beer, D., Buchert, L., Casey, E., DiMarco, R., \& Dolan, K. (1995) The effect of grammatical completeness on the communicative competence of students who use AAC. Paper Presented at the Annual Convention of the American Speech-Language-Hearing Association, Orlando, FL.

Todman, J. \& Alm, N. (1997). Talk boards for social conversation. Communication Matters, 11, $13-15$.

Vanderheyden, P. B. (1995a). Organization of prestored text in augmentative and alternative communication systems; an interactive schema-based approach. Technical Report \#AAC9501, Applied Science and Engineering Laboratories, University of Delaware, Newark, DE.

Vanderheyden, P. B. (1995b). An augmentative communication interface based on conversational schemata. Proceedings of the IJCAI '95 Workshop on Developing AI Applications for Disabled People, (pp 203-212). 